\title{
Frontières
}

\section{Étude sur le dit et le non-dit de la mort dans trois cartes de tarot}

\section{Danièle Bourque}

Volume 14, numéro 2, printemps 2002

La mort prononcée

URI : https://id.erudit.org/iderudit/1073964ar

DOI : https://doi.org/10.7202/1073964ar

Aller au sommaire du numéro

Éditeur(s)

Université du Québec à Montréal

ISSN

1180-3479 (imprimé)

1916-0976 (numérique)

Découvrir la revue

Citer cet article

Bourque, D. (2002). Étude sur le dit et le non-dit de la mort dans trois cartes de tarot. Frontières, 14(2), 14-19. https://doi.org/10.7202/1073964ar
Résumé de l'article

Cette étude porte sur le dit et le non-dit de la mort dans trois cartes de tarot : la carte de la mort du Tarot Colleoni du Victoria and Albert Museum de Londres, milieu du XV $\mathrm{XV}^{\mathrm{e}}$ siècle, la carte de la mort du Tarot parisien anonyme, première moitié du XVII ${ }^{\mathrm{e}}$ siècle, et la carte de la mort de l'Ancien tarot de Marseille de B. P. Grimaud, 1930. L'étude pointe en direction d'une manière d'agir la mort dans le langage. Elle se développe autour de la « la pensée de la parole et la parole en acte ", ce par quoi se conçoit la place qu'occupe la parole dans ces cartes sur la mort. 


\section{Résumé}

Cette étude porte sur le dit et le non-dit de la mort dans trois cartes de tarot: la carte de la mort du Tarot Colleoni du Victoria and Albert Museum de Londres, milieu du XVe siècle, la carte de la mort du Tarot parisien anonyme, première moitié du XVIIe siècle, et la carte de la mort de l'Ancien tarot de Marseille de B. P. Grimaud, 1930. L'étude pointe en direction d'une manière d'agir la mort dans le langage. Elle se développe autour de la « la pensée de la parole et la parole en acte", ce par quoi se conçoit la place qu'occupe la parole dans ces cartes sur la mort.

Mots clés : mort - tarot-rhéthorique parole-dit-non-dit.

\section{Abstract \\ This study concerns spoken and unspoken death within three death cards from the Tarot: that of the Tarot Colleoni from the Victoria and Albert Museum in London, middle of the 15th Century, that of the Anonymous Parisian Tarot, first half of the 17th Century, and that of the Marseille Tarot of the Ages by B. P. Grimaud, 1930. The study points to how death acts in language. Such means evolve around "language thought and language in action" whereby the role that language plays in death cards is devised.}

Key words: death - tarot - rhetoric speech - the unspoken.

\section{ÉTUDE SUR LE DIT ET LE NON-DIT DE LA MORT DANS TROIS CARTES DE TAROT}

\begin{abstract}
Danièle Bourque,
candidate au doctorat en sciences des religions, Département des sciences religieuses, UQÀM.
\end{abstract}

L'interrogation du dit et du non-dit de la mort dans le tarot requiert une discussion qui dépasse largement le cadre de cet article. Ces quelques lignes portent un projet plus modeste qui se limite à interroger la rhétorique générale de la mort dans le Tarot Colleoni, dans le Tarot parisien anonyme et dans l'Ancien tarot de Marseille. Par rhétorique, nous entendons, bien sûr, autre chose que cet «art de la persuasion » que désigne le mot dans son acception traditionnelle. La rhétorique désigne plutôt ici la condition générale de toute production discursive, s'agissant de la mort elle-même, sa mise en discours dans le jeu du tarot luimême. C'est à la lumière de l'expérience réfléchie de la parole, c'est sur la piste des manières d'agir la mort par le langage que sont examinées ici ces cartes de tarot.

Le jeu de tarot n'est donc pas, comme nous avons été habitués à le croire, porteurs d'un discours inspiré par la pensée hermétique. Les cartes ne sont pas envisagées ici comme des objets marqués au fer rouge de l'ésotérisme et forclos dans la marge du para-religieux, véhicules de données brutes sur la mort utilisés pour décrire la fin de la vie, pour évoquer son au-delà ou même pour formuler une annonce divinatoire de la mort. Comme nous le verrons, elles pointent en direction d'une manière d'agir la mort dans le langage.

Pourquoi une étude sur le dit et le nondit de la mort dans trois cartes de tarot? Texte $^{1}$ tout entier offert à l'interprétation, le tarot a, depuis le $\mathrm{XV}^{\mathrm{e}}$ siècle ${ }^{2}$, joué à dire ou à taire la mort. Ce jeu bien particulier du dit et du non-dit prend forme autour d'un seul atout, celui associé à la mort. Employer le mot «associé » ici pour désigner cette carte particulière relève d'une certaine prudence puisqu'au $\mathrm{XV}^{\mathrm{e}}$ siècle les atouts des tarots ne sont à peu près jamais numérotés ${ }^{3}$, ni légendés, leurs noms nous étant connus par différentes sources littéraires. On présume que la numérotation des lames, c'est-à-dire des cartes, aurait été introduite à différentes époques selon les traditions, mais qu'elle serait ultérieure au $\mathrm{XV}^{\mathrm{e}}$ siècle. L'apparition de légendes ${ }^{4}$ sur les cartes va néanmoins se multiplier grâce aux cartiers français à partir du XVII e siècle avec la production de jeux de tarots connus depuis lors sous le nom de Tarot de Marseille 5 . Or en créant les tarots de Marseille, les cartiers français ont légendé tous les atouts et toutes les figures à l'exception de la treizième lame, celle associée à la mort, qu'ils ont laissée sans nom. Et subséquemment, l'absence de mot pour désigner la mort fait école. Nombre de tarots qui viendront par la suite, calques de l'esprit ou de la lettre des tarots de Marseille, en font coutume. Mais cette coutume n'est pas une règle. Certains tarots légendent la carte de la mort, d'autres la laissent sans nom.

Pour peu que l'on s'y arrête, le dit et le non-dit de la mort dans le tarot ont tout pour étonner. Cet article interroge les manières inséparables qu'ont ces trois cartes de tarot de dire ou de taire la mort. Comment cherche-t-on à dire ou à taire la mort dans le tarot? Il importe d'aller audelà des lieux communs qui masquent l'essentiel dans l'expression « cartes de tarots sur la mort», afin de dégager ce qu'elle recouvre vraiment.

«Ich sterbe. Qu'est-ce que c'est? Ce sont des mots allemands. Ils signifient 
je meurs. Mais d'où, mais pourquoi tout à coup ? Vous allez voir, prenez patience. »(Sarraute, 1980, p. 11)

\section{LA THEORIA ET L'ERGON}

Devant nous trois cartes, enveloppes légères lestées de tout autre chose qu'elles. Quoi qu'elles donnent à voir, ces cartes du Tarot Colleoni, du Tarot parisien anonyme et de l'Ancien tarot de Marseille, se font transparentes, s'effacent pour mieux exposer ce qu'elles portent côté pile et côté face. Côté pile ${ }^{6}$, elles montrent qu'elles font partie d'un même jeu, qu'elles ne peuvent être mélangées à aucun autre. Côté face, elles isolent du monde tel objet, tel personnage, tel instant, telle époque pour les modaliser en signatures déictiques, les marquer en un afflux de démonstratifs : «ce», «celui », «ceci », « cela», «ça », etc.

Semblables à la photographie «qui emporte toujours son référent avec elle, tous deux frappés de la même immobilité amoureuse ou funèbre, au sein même du monde en mouvement» (Barthes, 1980, p. 17), ces cartes de tarots, bien avant de démonstrer ${ }^{7}$ la mort, se dissimulent jusqu'à nous faire oublier leurs enveloppes de papier-pâte et à nous empêcher de distinguer ce qu'elles représentent. Cette dissimulation, produite

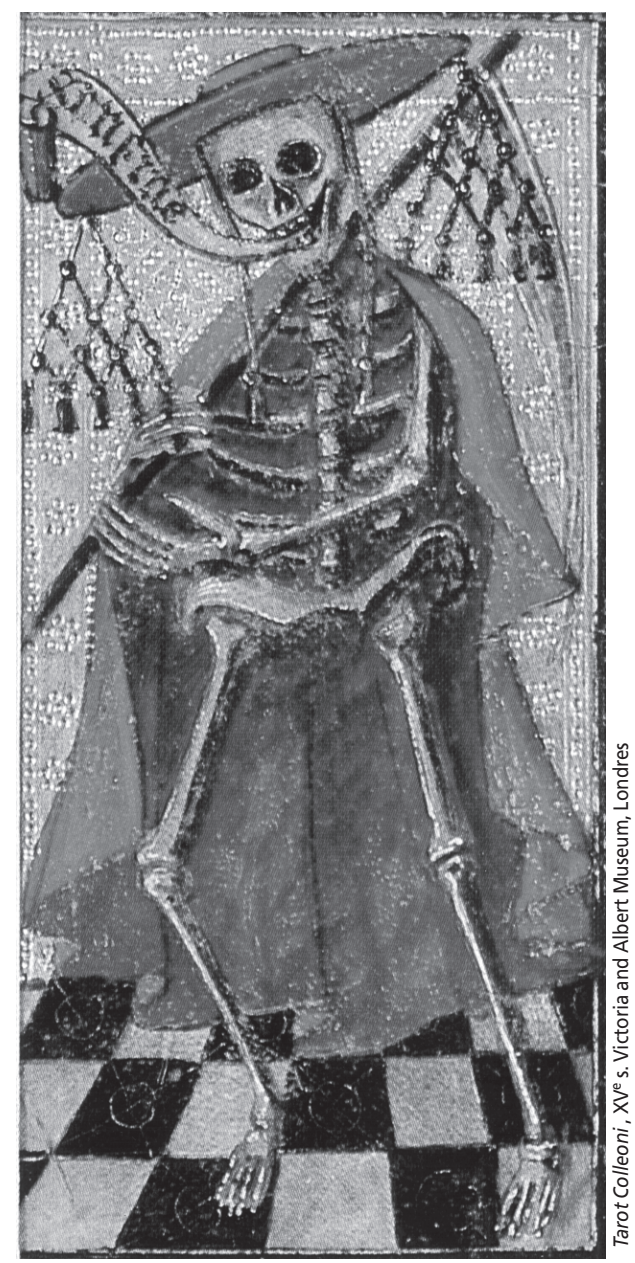

aussitôt que le regard se connecte à l'image, a pour cause le vis-à-vis intraitable avec le référent qui, tel un jeu de «dualités que l'on peut concevoir, mais non percevoir» (Barthes, 1980, p. 17), habite de part en part ces cartes de tarot.

Dans ces cartes, ce jeu dual prend la dimension d'un théâtre de réciprocités entre la theoria et l'ergon, "la pensée de la parole et la parole en acte» (Fumaroli et al., 1999, p. 2), abrités dans un même corps, le jeu de tarot. La theoria et l'ergon sont des figures de la parole, ce par quoi se conçoit la place qu'occupe la parole dans ces cartes sur la mort. Cette place ne se perçoit pas dans l'opacité immédiate des images, mais dans ce qui, dans ces trois cartes, tente d'interpréter la mort et de la dire, actualisant le verbe grec hermeneuein qui signifie à la fois « interpréter» et « parler». Theoria puisque ces cartes aménagent un habitat de la parole de la mort, présentent, chacune à leur manière, une conception de la mort. Ergon puisque que ces cartes ont toutes trois pour visée de mettre à nu, jusqu'à l'os, la part privée de toute mort, "cette zone d'espace, de temps, où je ne suis plus une image, un objet »(Barthes, 1980, p. 32), où je ne suis que depuis mon langage.

\section{LE TAROT COLLEONI: LA PAROLE SON FINE}

Depuis Antoine Court de Gebelin ${ }^{8}$, les tarots divinatoires foisonnent d'interprétations dites initiatiques de la mort («mourir pour renaître à une vie supérieure que confère l'initiation » ou encore "savoir mourir, est le grand secret de l'initié », etc.) où la mort-anéantissement est gage d'une renaissance, d'un nouveau cycle de vie. Si nous cessons un instant d'être captifs des interprétations caractéristiques de la "sémiosis hermétique» (Eco, 1992, p. 64) qui, dans ces cartes, voient l'annonce abrupte de la mort ou la révélation des secrets de l'audelà, nous découvrons une tout autre scène, celle de la pensée de la parole et de la parole en acte. Le Tarot Colleoni en est un exemple. Qu'est-ce à dire ? Dans cette carte, la mise en récit de la mort ne transmet pas l'expérience de l'anéantissement, mais pointe en direction d'un legs, dit ou spécifiquement tu (non-dit), là pour déjouer la ruine et du sujet et de son identité.

Tarot italien milieu du $\mathrm{XV}^{\mathrm{e}}$, le Tarot Colleoni naît dans un univers scolastique, chirographique et oral (disputatio universitaire, textes manuscrits, lecture à haute voix), dans la mouvance de cette crise qui, entre la fin du XIV et la première moitié $\mathrm{du} \mathrm{XV}^{\mathrm{e}}$ siècle, met en cause les « relations entre le langage et la réalité historique contemporaine, qui constitue un des aspects fondamentaux de la culture philosophique de ce temps»(Vasoli, 1999, p. 45). De nombreuses recherches montrent qu'au $\mathrm{XV}^{\mathrm{e}}$ siècle, le système des référents sémiotiques est complètement bouleversé. Les grandes disputes scolastiques, les querelles doctrinales bousculent les pouvoirs religieux et politiques mis à mal par de «nouvelles expériences intellectuelles et l'avènement d'autres formes d'organisation publiques»(Vasoli, 1999, p. 45). Le Tarot Colleoni, contemporain de cette crise, n'en est pas exempt. Il prend forme dans cet intervalle de libertés entre les vieilles lois et les nouvelles dont il est contemporain. Ce n'est donc pas un simple hasard si la mort, dans le Tarot Colleoni, accède à la pourpre. Attifé de la chape rouge des cardinaux, le squelette mime les plus hauts dignitaires de l'Église. Ceux-ci, du coup, paraissent grimés et funèbres, signe que leur pouvoir, remis en question, ne régit plus exclusivement les destinées. Ce squelette laisse échapper de sa bouche un phylactère sur lequel se lit son fine. Défi aux lois du probable et du possible, ces mots nous font entrer dans le domaine du surprenant: la mort parle! Mais pour nous surprendre, la mise en scène part du connu, le saisit dans sa course et le fige. Les pieds nus du squelette sur le sol carrelé, la chape rouge, le chapeau, la faux forment un ensemble reconnaissable où ce qui aurait pu être «n'importe quoi, devient alors le comble sophistiqué de la valeur» (Barthes, 1980, p. 60), celui de la parole son fine. La mort du Tarot Colleoni porte à l'existence la valeur d'une parole son fine. Cette parole dédouane toute limite. Dans sa volonté de tout dire, de ne pas supporter d'arrêt, la parole illimitée de « la mort » peut ainsi tout nommer, même ce qui ne peut l'être. Le peut-on?

Notre vie d'être parlant se déploie à l'intérieur d'un espace qui est celui que le langage lui assigne. Cet espace n'est pas infini. Il a des limites. Tout ne peut être dit, ce que dénie la mort du Tarot Colleoni pour en faire sa vertu cardinale. Ce qui ne peut être dit n'a rien à voir avec ce que veulent taire les conventions sociales ou ce que veut interdire la censure. On ne peut tout simplement pas sortir du langage. Wittgenstein l'a montré dans le Tractatus philosophicus : aux limites de notre langage correspondent les limites de notre monde. Wittgenstein ajoute que notre langage ne peut que décrire des faits. Quant aux valeurs et aux interrogations les plus essentielles, par exemple " qu'y a-t-il après la mort? », nous ne pouvons que nous plonger dans le silence: ce qu'on ne peut pas dire, enseigne Wittgenstein, il faut le taire. Il y a donc, pour le Wittgenstein du Tractatus, à élucider les conditions du discours valide et à percevoir les limites du langage. Dans la zone considérée valide de notre langage, les 
questions les plus fondamentales (le tropplein de sens) ne peuvent être exprimées. Elles se dissolvent d'elles-mêmes.

Nous assistons, depuis des décennies, au spectacle de cette dissolution montrée par des écritures du vide. Sorte de relevés des zones de vide, ces écritures tentent, par différentes pratiques langagières, de dépasser les limites du langage et se risquent, comme la mort du Tarot Colleoni, à nommer ce qui ne peut l'être, l'intangible, l'inaudible, l'innommable, l'indicible. Pensons aux recherches sur l'immatérialité de celui qui fut le chef de file du nouveau réalisme, Yves Klein. En 1958, après avoir vidé de ses objets usuels la galerie Iris Clert à Paris, puis repeint les murs en blanc et éclairé l'espace d'une lumière bleue, Klein expose le vide. Pensons aussi à cette œuvre musicale intitulée 4'33 (1952) du compositeur américain John Cage qui, durant quatre minutes trente trois secondes, fait entendre le vide. Pensons enfin à Martin Creed qui, à l'automne 2001, remporte le Turner Prize de la Tate Britain avec une installation intitulée The lights going on and off, lumières qui, au plafond d'une salle vide, s'allument et s'éteignent. Que veulent montrer ces écritures du vide?

Roland Barthes nous l'explique : « [...] dire je dors est en effet, à la lettre, aussi impossible que de dire je suis mort; l'écriture c'est précisément cette activité qui travaille la langue - les impossibilités de la langue - au profit du discours» (Barthes, 1984, p. 316). L'écriture cherche, dans le travail des impossibilités de la langue, à suppléer au défaut de l'appareil explicatif, à dire des espaces hors langage, à décrire ce qui échappe à toute description, et ce, dans une mise en cause du sujet du savoir au nom d'un autre sujet capable de dire l'indicible. Dans le Tarot Colleoni, la mort personnifie cet autre sujet en apparence capable de dire l'indicible. Mais quel est cet indicible?

Pour Wittgenstein, ce qui est indicible est logé au fondement du langage. Il distingue ainsi l'indicible qui se montre de ce qui se dit. Ce qui se dit relève du domaine de la science; cela concerne les états de choses descriptibles à travers le langage. À l'opposé, ce qui se montre concerne non pas le contenu dicible de la description mais le fait indicible de celle-ci, le fait du langage lui-même.

(Pierre, 1994, p. 17)

Or, dans le Tarot Colleoni, le recours à la mort qui parle recouvre de paroles le silence absolu de la mort jusqu'à l'emplir d'un «ce que je peux nommer ne peut me poindre»(Barthes, 1980, p. 84.). La mort

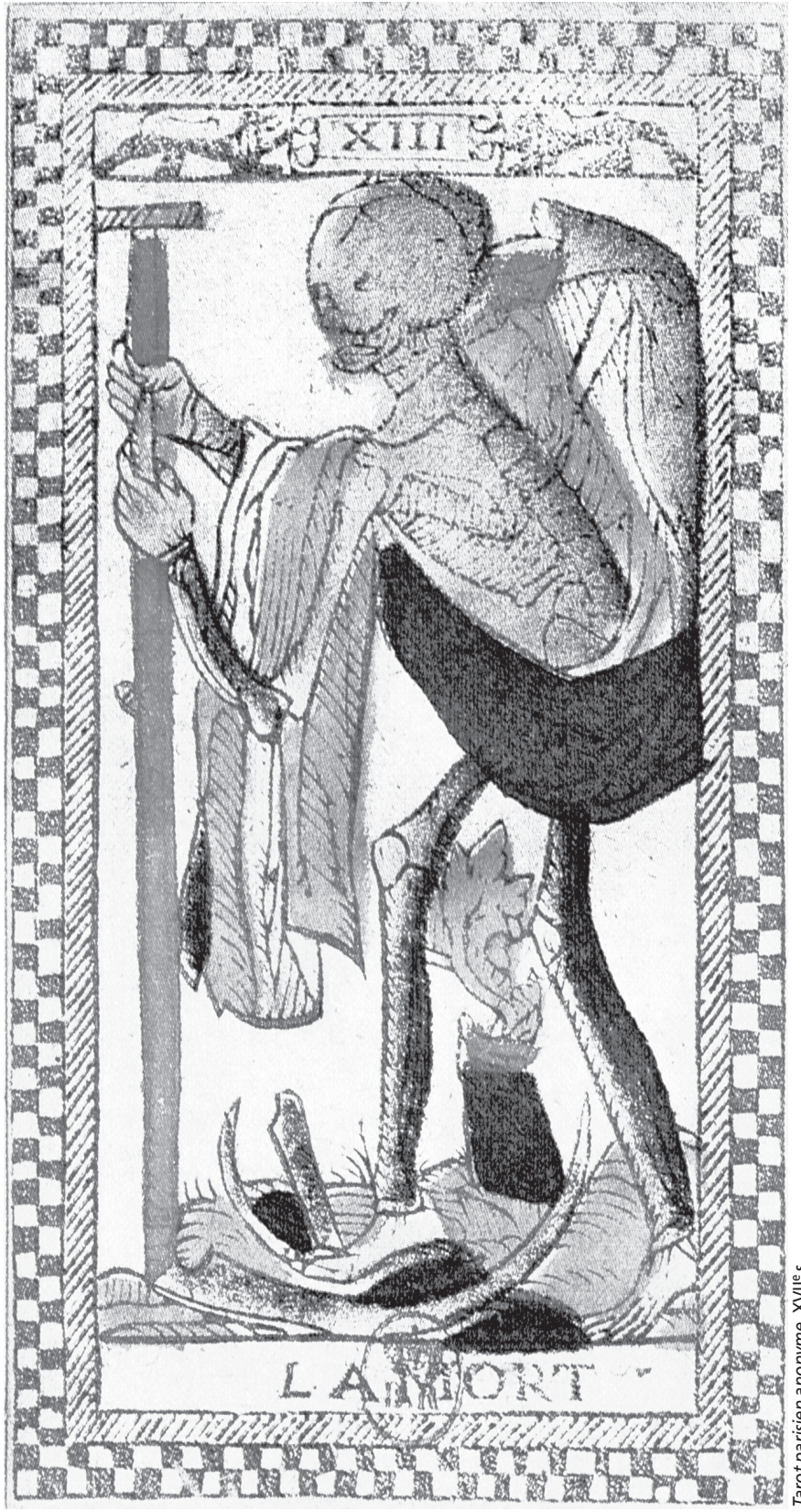

qui menace toute possibilité de dire et de survivre à l'extinction de la parole, au feu de sa radicalité son fine. Tant d'efforts parce qu'en présence du vide l'appareil explicatif tend à le remplir. Il ne s'agit pas d'une saturation ontologique du monde, d'un remplissage mécanique, mais plutôt d'un passage. Le Tarot Colleoni se fait en effet " passeur» qui, en voulant suppléer au vide pressenti de la mort, transmet la parole. Cette parole est transportée, éloignée de son habitat familier. Dans son dépaysement, elle n'est plus parole au sens propre mais au sens figuré. À cet instant, le Tarot Colleoni laisse voir sa fabrique de la mort qui consiste en une préparation heuristique des matériaux du discours dans le réseau de l'inventio, dans la rhétorique du convaincre et de l'émouvoir. L'enjeu est grand. Le squelette vêtu de sa chape d'apparat nous regarde les yeux vides, attend notre parole, seule condition pouvant l'accomplir. « $\mathrm{Au}$ pouvoir de la parole finale correspond un néant de la parole originelle; l'homme ne peut parler sans être accouché de sa parole, et pour cet accouchement il y a une technè particulière, l'inventio » (Barthes, 1985, p. 125). C'est ce que montre encore plus vivement le Tarot parisien anonyme.

\section{LE TAROT PARISIEN ANONYME: LA MATÉRIALISATION DE LA PAROLE}

Le Tarot parisien anonyme naît au $\mathrm{XVII}^{\mathrm{e}}$ siècle, à une époque où les traces de la présence humaine de chaque scriptor sont remplacées par des textes reproduits mécaniquement, où les objets imprimés deviennent des marchandises. Alors que «la Renaissance, préoccupée de rendre compte de l'harmonie entre l'homme et la Nature concevait les machines et les automates comme une magnification de la Création ", le $\mathrm{XVII}^{\mathrm{e}}$ siècle opère "un

qui parle oppose son son fine à la ruine du temps historique et biographique, se défend, dans le vif de la parole, à coup d'ergon, contre l'anéantissement, la déliquescence, le délabrement, l'effondrement, l'oubli. Le son fine se mue en pratique langagière qui, pour suppléer au défaut de l'appareil explicatif, en exalte les limites. Mais ne nous y trompons pas. Il ne s'agit pas de franchir les limites de la vie, mais d'emplir ce vide renversement de perspective mis en mouvement par un débat sur le statut théologique des machines » (Salazar, 1999, p. 792). Dans ce débat, les théologiens donnent voix à l'opinion qui résiste au mécanisme en se faisant gardiens de la tradition orale de l'Église et du Verbe incarné. Le XVII ${ }^{\mathrm{e}}$ siècle catholique est si marqué par la voix mystique qu'il s'y développe une sorte de physique spirituelle "à l'endroit de toutes les formes de matérialisation de la Parole sur- 
humaine, divine et diabolique, et dont la voix reste toujours l'organe privilégié » (Salazar, 1999, p. 810).

Le Tarot parisien anonyme laisse voir cette fascination pour les formes de matérialisation de la parole. On pourrait même supposer que ce tarot, issu de la lignée des tarots «de fantaisie» très appréciés au $\mathrm{XVI}^{\mathrm{e}}$ siècle, reste très proche de l'oralité. Il est important de souligner l'enracinement du tarot dans les rapports de vive voix. Qu'il soit support au simple jeu de cartes ou qu'il soit instrument de pratiques divinatoires, le tarot engendre un échange dans lequel la parole occupe une place centrale. En ce $\mathrm{XVII}^{\mathrm{e}}$ siècle où «le roi dialogue avec Dieu en présence des représentants de la parole publique puisque tel est le sens de «Parlement», ce corps auquel le roi délègue sa parole de justice et par qui le peuple parle» (Salazar, 1999, p. 813), où l'Église s'applique à la «physique» spirituelle de la voix, les tarots se font instruments de parole du peuple. Ils témoignent, dans un chassé-croisé d'influences religieuses, politiques et culturelles, de modes de sécularisation de la parole à l'âge classique.

Le Tarot parisien anonyme encadre la manifestation de la parole dans une suite de niches carrelées, serties de légendes et de chiffres qui éclairent sur le sens de chacune des cartes. Dans ce tarot français archaïsant à enseignes italiennes et à emprunts espagnols ${ }^{9}$, la carte XIII s'intitule clairement «La mort». Cette «mort» nous place à la frontière de la vie et de la mort. Sous nos yeux « le cadavre est vivant en tant que cadavre: c'est l'image vivante d'une chose morte», comme dirait Barthes (1980, p. 123), debout entre deux mondes, celui des vivants et celui des morts. Nous pourrions un instant nous croire dans le domaine $\mathrm{du}$ «ce n'est plus», mais nous sommes ailleurs, dans le «ça a été », où règne le refus de laisser la mort se transformer en deuil. Là aussi, comme dans le Tarot Colleoni, la carte de la mort du Tarot parisien anonyme ne transmet pas l'expérience de l'anéantissement. Il y a en elle un remplissage singulier, elle emplit de force la vue.

Mais à quel prix ? Celui de porter à vif, dans le deuil irrésolu, le manque. «Ce manque, en tant que manque, est impensable, pure absence, hiatus, syncope, anomalie, qui permet néanmoins à un être d'avoir son lieu» (Pierre, 1994, p. 21). Oui, mais pourquoi, par ce manque, la «mort» $\mathrm{du}$ Tarot parisien anonyme a-t-elle besoin d'un lieu? Barthes, se référant en cela à Aristote, écrit qu'un lieu, c'est «ce en quoi correspondent une pluralité de discours oratoires» (1985, p. 137). Un lieu, c'est aussi, toujours selon la lecture d'Aristote par

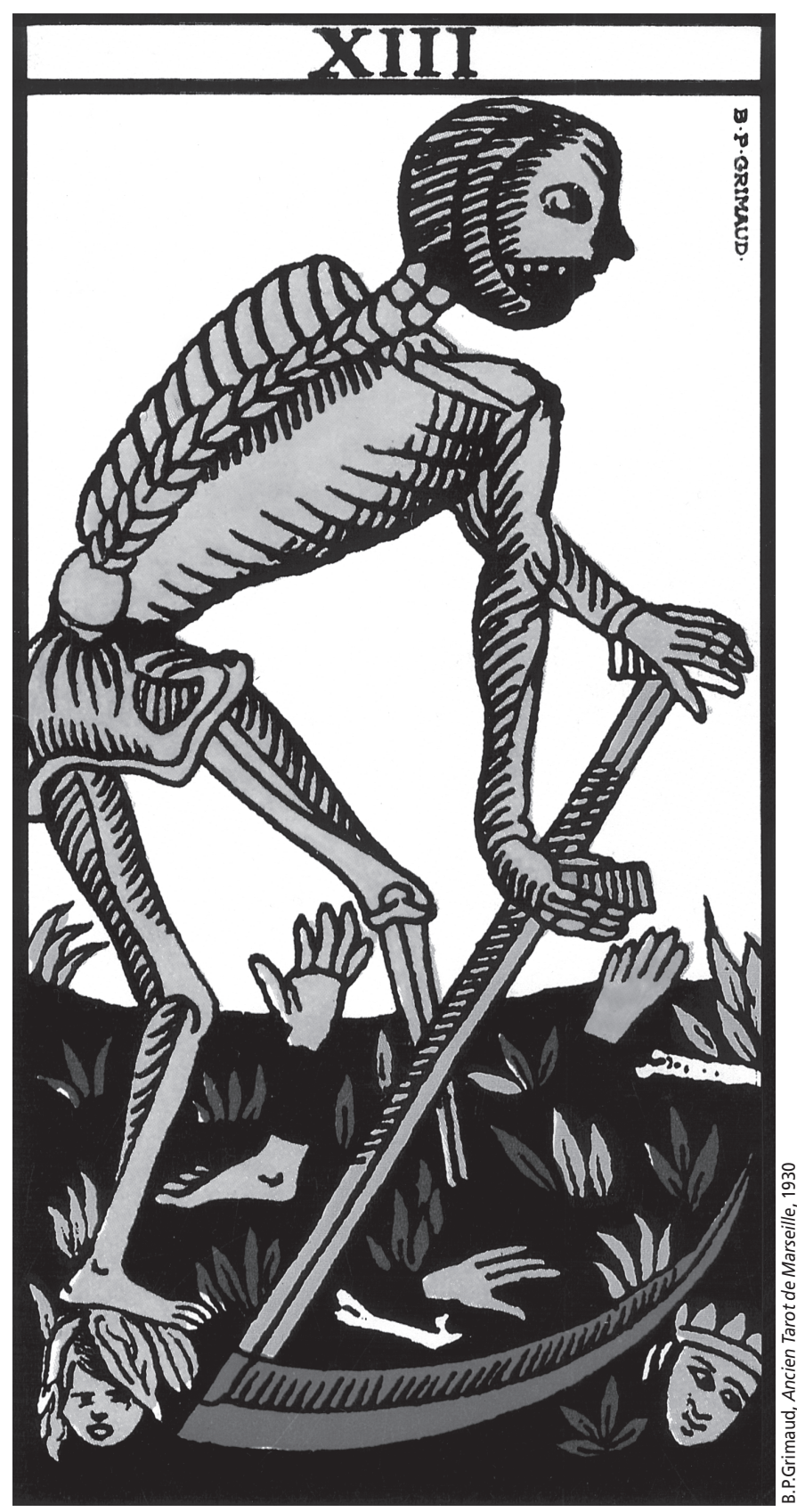

Barthes, «pour se souvenir des choses, il suffit de reconnaître le lieu où elles se trouvent (le lieu est donc l'élément d'une association d'idées d'un conditionnement, d'un dressage, d'une mnémonique); les lieux ne sont donc pas les arguments euxmêmes mais les compartiments dans lesquels on les range » (1985, p. 137). Qu'a voulu ranger le Tarot parisien anonyme dans ce lieu de la mort où erre le mort-vivant? La theoria, la pensée de la parole, sa représentation. Dans le Tarot parisien anonyme, la theoria est inventio, plus découverte qu'invention, "où tout existe déjà, il faut seulement le retrouver: c'est une notion plus extractive que créative » (Barthes, 1985, p. 125). Le manque ayant défini un lieu, le Tarot parisien anonyme, peut ainsi en extraire des arguments et les y ramener, «l'inventio est un cheminement (via argumentorum)» (Barthes, 1985, p. 125).

Ce cheminement prend forme dans une suite d'arguments développés au moyen de figures d'opposition et de substitution, supports au transfert de sens. Le personnage de toute évidence mort est vivant, ce qui est une antithèse. La mort et la vie sont côte à côte, dans un même corps au squelette dénudé et aux pieds et mains de chair, pareils à cette obscure clarté qui fait un oxymore. Cette manière de dire le mort superlativement vivant et le vivant superlativement mort est une hyperbole. La mort désigne la vie avec laquelle elle forme un ensemble dans un rapport d'appartenance, de nécessité propre à la synecdoque. La mort semble faucher la vie, telle une métaphore annoncée. Bref, tous ces arguments n'ont qu'un but: dire et redire la limite, conduire à cette frontière où il y a passage entre la vie et la mort, et non pas de la vie à la mort.

Ce passage, le Tarot parisien anonyme le condense à son plus haut degré d'intensité. Il le représente au moyen de l'image vivante d'une chose morte qui personnifie le continuum dans lequel toute vie se meut et nous emporte de la naissance à la mort. Ce continuum figure la vie entre la naissance et la mort. «La mort» du Tarot parisien anonyme ne traverse pas les frontières vers l'au-delà. Elle vit dans cet espace entre la vie et la mort, enjambe les seuils, comme autant de petites morts, que nous franchissons à différents âges de la vie. "La mort» entre ainsi dans le domaine dicible et, se matérialisant, peut être nommée. «La nomination est l'affaire du discours universel, du discours commun à tous » (Descombes, 1997, p. 47). Ce discours commun sur la mort, le Tarot parisien anonyme le fait éclore en moi, en tel lieu, tel moment, tel instant de la traversée d'un seuil d'existence. La mort dit ce qui en moi dit nous et ce qu'elle dit vaut pour tous. 
Dans le tête-à-tête que propose le Tarot parisien anonyme avec la mort, il n'y a pas, derrière, de secrets sur la vie après la mort comme le voudraient des interprétations caractéristiques de la sémiosis hermétique. Il y a plutôt, devant, dans la matérialisation de la parole, une proposition d'existence: s'exposer à ce qui se déploie, se découvre, se révèle. La mort du Tarot parisien anonyme appelle à la découverte, à l'effort de comprendre où "comprendre, c'est reconnaître que l'on n'a jamais assez compris, c'est reconnaître que toutes les significations demeurent en suspens tant que l'on n'a pas achevé de se comprendre soi-même, de se laisser déchiffrer par la médiation des mots» (Rochais, 1994, p. 141).

\section{L'ANCIEN TAROT DE MARSEILLE : LA PAROLE DÉRÉELLE}

Il n'en va pas ainsi de la mort dans l' $A n$ cien tarot de Marseille de B. P. Grimaud. En 1930, la Société Grimaud (aujourd'hui France Cartes), célèbre fabricant de cartes à jouer, publie l'Ancien tarot de Marseille. C'est Paul Marteau, petit neveu de BaptistePaul Grimaud, qui incite la Société à remanier le Tarot "italien» de Grimaud (1890) et le Tarot "italien» de Lequart (1891). Ces tarots à enseignes italiennes auraient vraisemblablement fait partie $d u$ fonds de l'atelier du maître cartier Arnould, atelier racheté par Grimaud en 1848. Dans ces tarots fidèles au modèle du Tarot de Besançon $^{10}$, Paul Marteau remplace Junon et Jupiter par la Papesse et le Pape des tarots de Marseille. Marteau légende tous les atouts et toutes les figures à l'exception de la mort qu'il laisse sans nom, comme dans les tarots de Marseille.

Théâtre du non-dit, la carte de la mort de l'Ancien tarot de Marseille est theoria sans mot, ergon que nous ne pouvons dire. «Du non-dit comme rien à dire, il n'y a grand-chose à ajouter, semble-t-il, sinon que je trouve en lui ma limite, limite de ma capacité à parler comme mon appétit pour tous les lieux (ou topoi) ainsi exclus de mon parcours discursif» (Descombes, 1997, p. 17). Conduits à la limite de notre capacité à parler, la carte de la mort nous montre, sans mot, que nous ne pouvons la dire, nous fait un signe, un "pas un mot», comme si quelqu'un pouvait entendre.

Mais qui écoute? Tel autre, non pas quelqu'un «mais ce lieu vers lequel je dirige et ordonne mon propos» (Descombes, 1997, p. 35). Quel est ce lieu? Celui de la topique: une méthode; une grille de formes vides; une réserve de formes remplies. Méthode parce que le recours à une mort sans mot représente, dans l'Ancien tarot de Marseille, un moyen court et facile "pour trouver la manière de discourir même sur les sujets qui sont entièrement inconnus » (Barthes, 1985, p. 138). Cette méthode consiste à nous mettre en état "sur tout sujet proposé, de fournir des conclusions tirées de raisons vraisemblables » (Barthes, 1985, p. 138). Grille de formes vides puisque la carte de la mort, laissée sans mot, tient lieu de case vide. Lorsque j'entre en contact avec cette case vide "surgit une idée possible, une prémisse d'enthymème » (Barthes, 1985, p. 138), l'enthymème étant un cheminement qui me donne le sentiment de découvrir quelque chose de nouveau «par une sorte de contagion naturelle, de capillarité qui étend le connu (l'opinable) vers l'inconnu » (Barthes, 1985, p. 132). Réserve de formes remplies enfin, puisque « les lieux sont en principe des formes vides; mais ces formes vides ont tendance à se remplir toujours de la même manière, à emporter des contenus, d'abord contingents, puis répétés, réifiés »(Barthes, 1985, p. 139).

Le lieu de la mort de l'Ancien tarot de Marseille n'est rien d'autre qu'une réserve de stéréotypes, un amalgame de conceptions de la mort prêtes-à-dire comme autant de «morceaux pleins que l'on place obligatoirement dans tout sujet» (Barthes, 1985, p. 139). Paul Marteau, qui destine son tarot aux cartomanciennes, accompagne le jeu d'un livret dans lequel il présente «la mort ${ }^{11}$ d'abord comme «symbole du mouvement et de la marche progressive qui récolte au moyen de la faux » (Marteau, 1969, p. 31), puis, littéralement, comme « la mort pris dans son sens courant, l'arrêt de quelque chose, l'immobilité » (Marteau, 1969 , p. 31 ). N'oublions pas que nous sommes en 1930 et non au XVII ${ }^{\mathrm{e}}$ siècle, époque de la création des premiers tarots de Marseille. L'interprétation de la mort que présente Marteau demeure semblable aux interprétations que véhiculent par exemple le Tarot des bohémiens de Papus (1889), le Tarot Waite d'Arthur E. Waite (1910), le Tarot des imagiers du Moyen Âge d'Oswald Wirth (1926). Ces tarots présentent des interprétations de la mort communes au grand courant occultiste inauguré à la fin du XVIII ${ }^{\mathrm{e}}$ siècle par Éliphas Lévi, pseudonyme hébraïsant d'Alphonse-Louis Constant, qui a introduit le tarot dans le courant cabalistique de l'occultisme français. Chez Lévi, les cartes deviennent des " clés », des « arcanes » porteurs d'une connaissance secrète provenant, selon Lévi, d'une Tradition transmise depuis des siècles, d'initiés en initiés. Adepte du "canon» occulte, Marteau répète, réifie cette idée de la mort qui, depuis Lévi, signifie la fin d'un cycle, la mort d'un état pour mieux renaître à un autre. Marteau fige cette idée de la mort telle "une croyance fondamentale, une Urdoxa que rien ne peut défaire» (Barthes, 1980, p. 165). Autrement dit, dans
l'Ancien tarot de Marseille, la mort est en arrêt d'interprétation.

Cette mort est figée sur la table des choses, recouverte d'un sans mot saturé de réassurances ésotériques. Ce «sans mot» qui élimine toute possibilité d'investir l'imaginaire appartient à un monde déréel, tel un trou noir de la relation à l'ergon. Dans un monde «irréel (je le parle différemment)» (Barthes, 1977, p. 106). Dans un monde «déréel (je le parle avec peine)» (Barthes, 1977, p. 106). Alors que l'irréel se dit avec abondance dans la fiction, «le déréel ne peut se dire; car si je le dis (si je le pointe, même d'une phrase malhabile ou trop littéraire), c'est que j'en sors » (Barthes, 1977, p. 107).

On pourrait dire, paraphrasant en cela Michel Foucault ${ }^{12}$, que dans l'Ancien tarot de Marseille, la mort est une idée en train de disparaître. Ce qui s'amuiit, s'efface, échappe à la vue, à l'image de la proscription de la lettre E dans La Disparition, roman de Georges Perec, c'est une disposition à tolérer le vide, à séjourner dans l'épaisseur du symbolique ${ }^{13}$, à vivre le réel ${ }^{14}$, dans ce qui n'a pas encore de forme, dans ce qui reçoit du symbolique la possibilité de se manifester.

\section{LA PLACE VIDE}

Dire la mort, l'annoncer, la recevoir, la raconter et même la taire, engendre l'expérience d'une place vide qu'accessoirement les savoirs tentent de combler et, autour de laquelle se déploie, comme dans le cas du religieux, une communauté d'affects. Cette place vide nous la connaissons, elle loge dans notre parole. Elle se montre lorsque, par exemple, l'annonce de la mort nous parvient, provoque une coupure, interrompt le cours de la locution, nous laisse "sans mot » du côté du « je ne sais pas quoi dire», dans la brisure de l'expression, balbutiant un retour à la parole.

Cette place vide, nous l'avons rencontrée dans l'Ancien tarot de Marseille. La côtoyer nous a permis de pressentir que les cartes de la mort du tarot de Marteau, du Tarot Colleoni et du Tarot parisien anonyme sont des dispositifs lacunaires, subtilement agencés pour faire circuler, dans le jeu de tarot, une case vide. À l'entrée, ces trois cartes présentent des arguments sur la mort. Ce qu'on trouve à la sortie, ce sont des cases vides, «de la place sans occupant et de l'occupant sans place» comme dirait Deleuze (1969, p. 55). Il n'y a pas dans ces cartes de «hors case » où la mort pourrait être éjectée une fois pour toutes. Il n'y a qu'une case vide, celle de la mort, semblable à la case vide du jeu de taquin. Ce jeu se présente comme un carré (le plateau du jeu) à l'intérieur duquel coulissent des petits carrés. Le jeu consiste à déplacer ces carrés 
vers la «case vide», horizontalement ou verticalement, de manière à recréer l'image globale du casse-tête que contient le jeu. Dans les trois cartes étudiées, la mort tient lieu de "case vide». Cette case vide ne s'imagine pas comme un champ clos, mais comme un «non-lieu, une pure distance», dirait Foucault (2001, p. 1012), interstice, point de surgissement des événements pour que ceux-ci s'inscrivent sur la surface des choses et qu'ensuite, le langage les marque et les idées les dissolvent (Foucault, 2001, p. 1011). «Une case vide qui n'est ni pour l'homme ni pour Dieu [...] tout cela traversé par des échos, des circulations, des échos des événements qui font plus de sens et de liberté et d'effectivité que l'homme n'en a jamais rêvé, ni Dieu conçu» (Deleuze, 1969, p. 91). En elle, case vide, existent à la fois l'impersonnalité du mourir «le moment où je me perds hors de moi » et «le moment où la mort se perd en ellemême» (Deleuze, 1969, p. 179). Ces moments précis, ceux qui ont été témoins de la mort les connaissent bien. La mort qui vient de se produire devient «la figure singulière que prend la vie la plus singulière pour se substituer à moi » (Deleuze, 1969, p. 179). Autrement dit en elle, place vide et toujours mouvante, prennent forme des manières d'agir la mort par la parole comme autant de fragments de théoria et d'ergon qui, au contact les uns des autres, ne cessent de se déployer, de s'agiter, de se mouvoir, toutes forces tournées vers cette question: que tenons-nous pour certain?

\section{Bibliographie}

BARTHES, Roland (1985). L'aventure sémiologique, Paris, Seuil.

BARTHES, Roland (1984). Le bruissement de la langue, Paris, Seuil.

BARTHES, Roland (1980). La Chambre claire, Paris, de l'Étoile.

BARTHES, Roland (1977). Fragments d'un discours amoureux, Paris, Seuil.

DEPAULIS, Thierry, (1984). Tarot, jeu et magie, Paris, Bibliothèque nationale de Paris.

DELEUZE, Gilles (1969). Logique du sens, Paris, Éditions de Minuit.

DESCOMBES, Vincent (1997). L'inconscient malgré lui, Paris, Éditions de Minuit.

ECO, Umberto (1992). Les limites de l'interprétation, Paris, Grasset \& Fasquelle.

FOUCAULT, Michel (2001). Dits et écrits 1, 1954-1975, Paris, Gallimard.

FOUCAULT, Michel (1966). Les mots et les choses, Paris, Gallimard.

FUMAROLI, Marc (dir.) (1999). Histoire de la rhétorique dans l'Europe moderne, 1450-1950, Paris, Presses universitaires de France.
GREIMAS, Algirdas Julien et Teresa Mary KEANE (1992). Dictionnaire du moyen français. La Renaissance, Paris, Larousse.

MARTEAU, Paul (1969). Ancien Tarot de Marseille. B.P. Grimaud. Paris, Éditions J.-M. Simon.

PAPUS (1971). Le Tarot des bohémiens, Paris, Éditions Dangles.

PIERRE, Jacques (1994). "Limpasse de la définition de la religion: analyse et dépassement», Religiologiques, ${ }^{\circ}$ 9, printemps, p. 15-29.

ROCHAIS, Gérard (1994). «Du bricolage exégétique », Religiologiques, $\mathrm{n}^{\circ} 9$, printemps, p. 139-155.

SARRAUTE, Nathalie (1980) L'usage de la parole, Paris, Gallimard.

SALAZAR, Pierre-Joseph (1999). «La voix au XVII ${ }^{\mathrm{e}}$ siècle », dans Marc Fumaroli (dir.) (1999). Histoire de la rhétorique dans l'Europe moderne, 1450-1950, Paris, Presses universitaires de France, p. 787-821.

THOMAS, Louis-Vincent (1991). «La mort aujourd'hui : de l'esquive au discours conve$\mathrm{nu}$ », Religiologiques, $\mathrm{n}^{\circ}$ 4, p. 17-43.

VASOLI, Cesare (1999). "L'Humanisme rhétorique en Italie au $\mathrm{XV}^{\mathrm{e}}$ siècle», dans Marc Fumaroli (dir.) (1999). Histoire de la rhétorique dans l'Europe moderne, 1450-1950, Paris, Presses universitaires de France, p. 45-129.

WIRTH, Oswald (1978). Le Tarot des imagiers du Moyen Âge, Paris, Tchou.

WITTGENSTEIN, Ludwig (1961). Tractatus logico-philosophicus; suivi de Investigations philosophiques, Paris, Gallimard.

\section{Notes}

1. «Partout où une activité signifiante est mise en scène selon des règles de combinaison, de transformation et de déplacement, il y a du texte» (Barthes, 1984, p. 102).

2. L'état actuel de la recherche sur le tarot montre qu'il n'y a aucune certitude sur l'origine de ces cartes. Si les premiers témoignages de l'existence des cartes à jouer en Europe émergent et se multiplient autour des années 1370, nous n'avons jusqu'ici retrouvé la trace de l'apparition du tarot qu'à partir du deuxième quart du $\mathrm{XV}^{\mathrm{e}}$ siècle, moment où apparaissent des cartes appelées triomphe, ou trionfi, ou triumphorum ludus, nom ancien du tarot.

3. Notons cependant que dans le tarot de Charles VI, tarot de l'Italie du Nord de la fin du $\mathrm{XV}^{\mathrm{e}}$ siècle, des chiffres sont inscrits sur certains atouts. Ces chiffres romains tracés à l'encre paraissent avoir été ajoutés après la réalisation des cartes, au plus tard vers la première moitié du $\mathrm{XVI}^{\mathrm{e}}$ siècle.

4. Des légendes existent déjà sur les Tarots de Mantegna, série d'estampes qui ne sont ni tarots ni œuvre de Mantegna. Ces exemples de la gravure ferraraise de la deuxième moitié du $\mathrm{XV}^{\mathrm{e}}$ siècle, proches des allégories du tarot, sont numérotés en chiffres romains et arabes et légendés en dialectes voisins de ceux de Venise et de Ferrare. Les cartiers français qui produisent au $\mathrm{XVI}^{\mathrm{e}}$ siècle et $\mathrm{XVII}^{\mathrm{e}}$ siècle des tarots d'influence très italianisantes ont subi l'attraction de modèles italiens et dans certains cas ibériques.

5. Le Tarot de Marseille, qui vraisemblablement n'aurait pas été introduit par les cartiers marseillais, puise ses origines à Milan. Il «désigne un modèle bien particulier de tarot français à enseignes italiennes, dont la diffusion, sous une forme stéréotypée, semble avoir été très grande. Aujourd'hui réservé aux cartomanciennes, ce type de cartes a été d'un usage courant parmi les joueurs français jusqu'au début du $\mathrm{XX}^{\mathrm{e}}$ siècle» (Depaulis, 1984, p. 71).

6. On trouve au dos du Tarot Colleoni un fond uni, au dos du Tarot parisien anonyme des hexagones avec «Croix de Malte» et au dos de l'Ancien tarot de Marseille des motifs hélicoïdaux.

7. Démonstrer: Exposer, expliquer (Greimas et Keane, 1992).

8. Antoine Court de Gebelin, pasteur protestant et franc-maçon, publie, en 1781, le huitième volume du Monde primitif, ouvrage considéré par certains comme l'initiateur de la première vague d'utilisation du tarot comme outil divinatoire. Il faut cependant faire mention d'un ouvrage plus ancien qui traite de cartomancie et de divination par les cartes, Le Sorti di Francesco Marcolino da Forli, intitolate Giardino di Pensieri allo Illustrissimo Signore Hercole Estense, Duca di Ferrara, publié à Venise en 1540.

9. Les as sont ornés d'animaux fantastiques inconnus des jeux italiens mais assez fréquents dans les cartes ibériques.

10. Le Tarot de Besançon, tarot du XVII ${ }^{\mathrm{e}}$ siècle, est une variante du Tarot de Marseille, où la Papesse et le Pape ont été remplacés par Junon et Jupiter.

11.Il faut noter que la lame XIII, qui n'est pas légendée dans le jeu, est titrée «la mort» dans le livret.

12. Dans Les mots et les choses, Michel Foucault postulait que l'homme est une idée en train de disparaître (Foucault, 1966).

13. Le symbolique, à ne pas confondre avec la symbolique, la faculté de symboliser, les symboles. Terme introduit par Lacan, le symbolique désigne l'ordre des phénomènes qui, en cure psychanalytique, sont structurés comme un langage. C'est aussi, "le registre de la substitution et du manque qui implique non seulement ce qui est de l'ordre du manque et de la perte, mais le consentement au manque, à la perte et à la séparation » (Thomas, 1991, p. 18).

14. Le réel fait référence ici à cette forme d'existence psychique qu'il ne faut pas confondre avec la réalité matérielle, la mise en forme du réel. 